\title{
Is wavefront coding an alternative to adaptive optics for retinal imaging?
}

\author{
E.Acosta, J.Arines, C. Almaguer-Gómez \\ Física Aplicada, Universidade de Santiago de Compostela \\ Santiago de Compostela, Spain \\ eva.acosta@usc.es
}

\author{
S. Bosch and S. Vallmitjana \\ Dept. Física Aplicada, Universitat de Barcelona \\ Barcelona, Spain
}

\begin{abstract}
In this work we explore the use of the wavefront coding technique for obtaining high resolution images of the retina of human eyes. The numerical analysis pretends to show the robustness of the technique under changes in pupil diameter and wavefront shape including intersubject and intrasubject variability, using always the same restoration filter or image decoder. The results show that it is possible to obtain high resolution images under different ocular aberrations and pupil diameters with the same decoder, opening the possibility of real time high resolution images with this low-cost and robust technique.
\end{abstract}

Keywords-Wavefront Coding; Retinal images; Ocular aberrations; Deconvolution; Adaptive Optics

\section{INTRODUCTION}

High resolution images of the retina are important for early diagnostic of ocular pathologies or following the therapeutic progress of pharmacological treatments, among other interests. But retinal images are degraded by the presence of ocular aberrations. These aberrations change with time and between subjects. During the last decades different techniques have been proposed to overcome the effects of the aberrations. Adaptive Optics [1], Deconvolution from wavefront sensing [2], Blind Deconvolution [3] and Wavefront Coding [4,5] are the most representative examples. Adaptive Optics (AO) is the most used technique to obtain quality images of the retina at different levels of detail. It is a pure optical technique that provides very good results by compensating aberrations prior to image recording what increases significantly the signal to noise ratio of the images and avoids the spurious artifacts typical of deconvolution process. Its main drawbacks are its complexity and cost. The technique of Deconvolution from Wavefront Sensing (DWFS) was proposed as a low cost competitor to AO. It can be classified as a hybrid technique that uses optical information (the wavefront sensor for calculating the impulse response of the system) and a digital procedure (deconvolution) to restore the degraded image. Its main drawback is the low signal to noise ratio (SNR) of the images due to the blur induced by the optical aberrations. In order to increase the SNR it is possible to average different images or correct the static aberrations of the eye what gives rise to the technique known as Partially Compensated Deconvolution from Wavefront Sensing (PCDWFS) [6]. However this technique presents the problem of needing a phase mask for each subject in order to compensate the static aberrations of the eye. Recently, some of the authors of this work [7] suggested a different approach: instead of compensating the static aberrations, huge amount of a known aberration is included in the optical system and the impulse response is nearly invariant to changes in the ocular aberrations. This is the basis of the Wavefront Coding (WFC) technique applied to retinal imaging. Due to the fact that the impulse response is nearly invariant and the OTF of the system has no zeros, it is possible to use the same restoration filter (or image decoder) for restoring the images obtained from different subjects or the same subject but at different moments in time.

\section{WAVEFRONT CODING}

Wavefront Coding is a technique proposed in the 90s by E. Dowski and W. Cathey [4]. This technique involves a wavefront coding element which is an optical element or system such that the optical transfer function of the system is substantially invariant to focus-related aberrations from the intervening medium. WFA involves a detector that records the coded image, and a postprocessing step for image decodification. This technique has been extensively used, for instance, in surveillance camera systems in order to obtain sharp images at different depths and with moving objects. In Fig. 1 we show a scheme of the WFC technique.

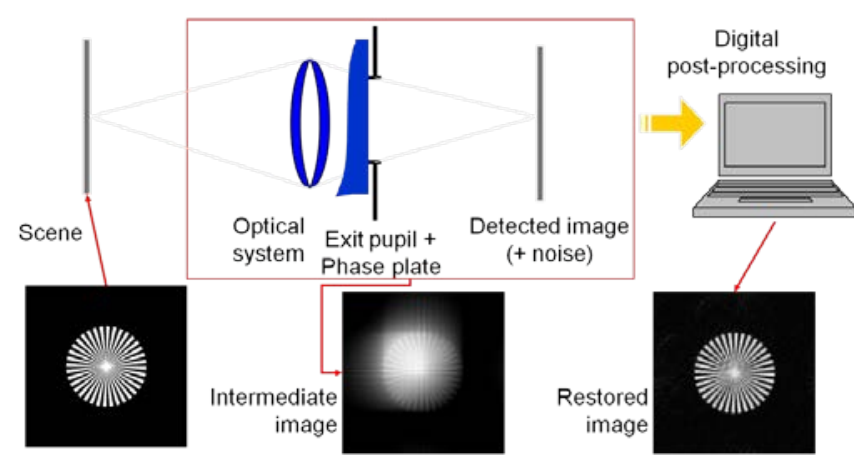

Fig. 1. Scheme of the wavefront coding technique

We must stress the fact that for the case of high resolution images of the human retina, WFC has to face not only with 
defocus but also with astigmatism and higher order aberrations. Moreover, the eye exerts changes in the pupil size depending on the target luminance, the subject and drugs delivered.

\section{ROBUSTNESS OF WAVEFRONT CODING}

In the previous section we introduced the sources of problems to obtain high resolution imaging through the human eye: Different amounts of defocus, astigmatism and higher order aberrations, as well as changes of pupil size occur during retinal imaging either for a specific subject or for a population.

In order to analyze the robustness of WFC in presence of changes of ocular aberrations and pupil size, we conducted numerical simulations to evaluate the performance of the technique. We depart from a simplified eye model with a lens with a focal length of $16.6 \mathrm{~mm}$. We generate random pupil radius values from a gaussian distribution with $3.0 \mathrm{~mm}$ mean and a standard deviation of $0.2 \mathrm{~mm}$. Two different sets of ocular aberrations were used:

A) 21 sequences of aberrations of subject JA measured with a Shack-Hartmann wavefront sensor during 15s;

B) 10 sequences of ocular aberrations randomly generated by using the statistical model proposed by Thibos et.al. [8].

We used the USAF test as object. For the codification of the wavefront we used a cubic phase in the shape $30 \lambda\left[\left(\mathrm{x}^{3}+\mathrm{y}^{3}\right)\right.$ $3\left(x^{2} y+y^{2} x\right)$ ) (where $x$ and $y$ are normalized coordinates to $3 \mathrm{~mm}$ radius). In order to show the degradation of retinal images due to eye aberrations, we performed simulations of the image of the test in the focal plane of the simplified eye in presence of the ocular aberrations. In order to obtain the image for the post processing we also simulated the images in presence of the ocular aberrations and those of the cubic phase mask. From here all images are restored with the same Wiener filter for the PSF corresponding to the cubic phase plate for a pupil radius of $3 \mathrm{~mm}$.

\section{RESULTS}

As explained above, we will group the results for the dynamic correction of the retinal images of a single subject and for a static one of 21 subjects.

\section{A. Temporal Variability}

In this case we include the a priori correction of the mean value of low order aberrations (about $80 \%$ ), in order to mimic the situation where the operator compensates the mean defocus and astigmatism before the picture of the retina is taken. In Fig. 2 we show the variability of the pupil radius included in the simulation of the 21 temporal frames.

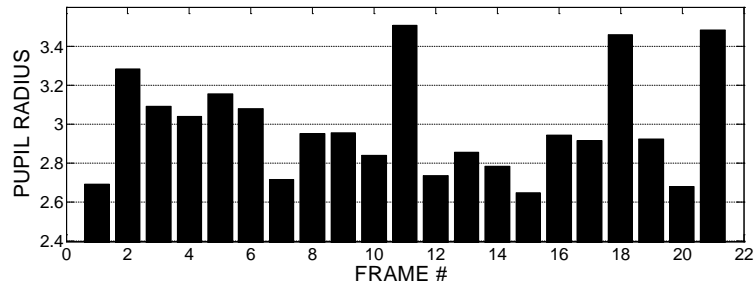

Fig. 2. Pupil radius used for generating the different images of the temporal serie.

In Fig. 3 we present two images of the stack (\#1 \& \#7) blurred by the aberrations of the eye at two different recording times. We can observe that the small time-dependent changes in defocus and astigmatism in combination with changes of the high order aberrations cause significant changes in the visibility and detectable details of the USAF chart.

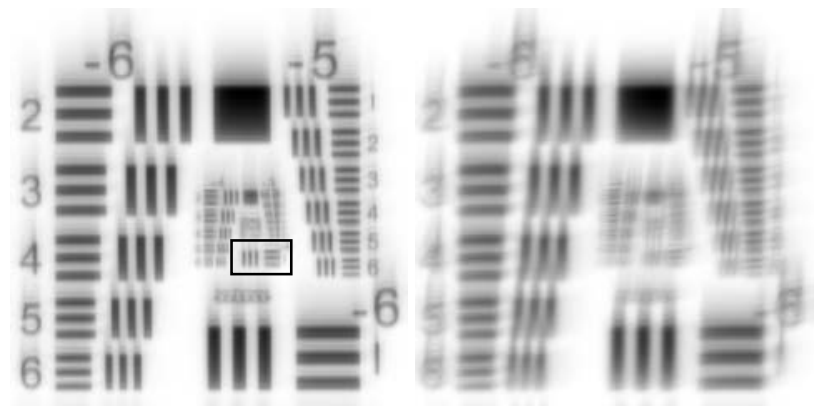

Fig. 3. Two frames of a sequence of images degraded by the temporal changes in the aberrations of one eye. Each line of the black rectangle has a thickness of $2.5 \mu \mathrm{m}$.

In Fig. 4 we show the restored images* applying the WFC technique for the same frames as in Fig. 3 (*Restored from the images blurred by the eye aberrations and the cubic phase plate). For conciseness, focusing our attention in the 3 bar patterns inside the marked rectangle, we conclude that they are both detectable.

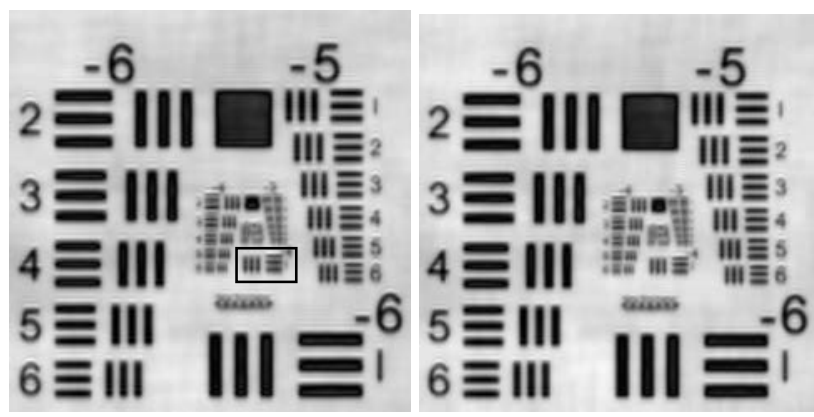

Fig. 4. Restored images with WFC technique corresponding to the images of figure 2. Each line of the black rectangle with a thickness of $2.5 \mu \mathrm{m}$ is detectable in both.

\section{B. Population variability}

In this section we evaluate the performance of WFC when retrieved images correspond to different subjects and therefore to different ocular aberrations and pupil sizes. We consider 
again a partial a priori compensation (about 80\%) of the low order aberrations.

In Fig. 5 we show two images of the sequence with two different aberrations and pupil diameters.

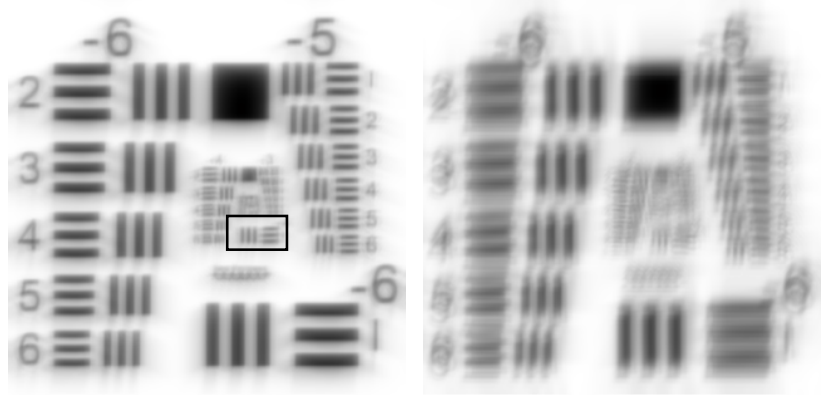

Fig. 5. Two frames of a sequence of images degraded by the aberrations of two different eyes. Each line inside the black rectangle has $2.5 \mu \mathrm{m}$.

In Fig. 6 se show the restored images obtained with the same restoration filter, built considering just the WCF codification phase and a pupil radius of $3 \mathrm{~mm}$.

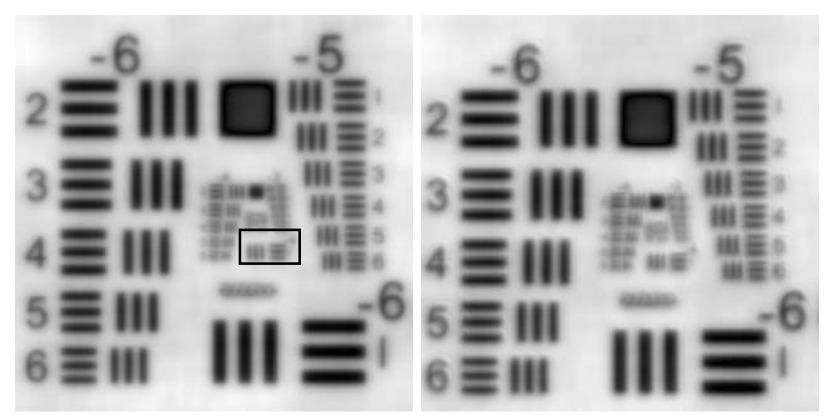

Fig. 6. The restored images corresponding to the images presented in fig 5 . Each line inside the black rectangle has $2.5 \mu \mathrm{m}$.

Finally we also tested the behavior of WFC in presence of noise in the recorded images. We generate several images by adding a random gaussian noise, providing a SNR of 23. In Fig. 7 we illustrate the results for the image corresponding to Fig.3 (left). Although some spurious structures appears the achieved resolution is comparable to the noise-free case.

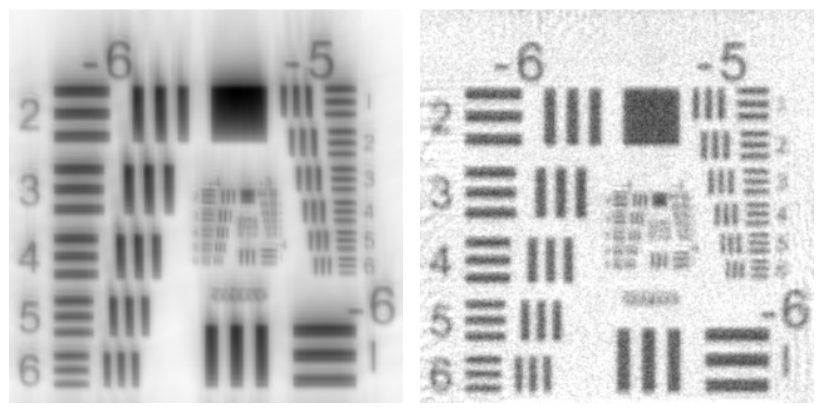

Fig. 7. Example of a degraded and restored images in presence of noise

\section{DisCussion}

The main limitation for obtaining high resolution images of the retina are the changes of the ocular aberrations between subjects, and even for each subject due to their temporal variation. AO provides high quality images but the optical systems are complex and expensive. Different techniques have proven their suitability and their drawbacks. For those techniques that rely on image postprocessing as DWFS or WCF the size of the pupil is also an important parameter if the limiting aperture is the eye pupil, because the eye pupil changes also between persons and time. In this work we have shown that WFC can handle the changes of both ocular aberrations and pupil size, using the same image restoration filter (or image decoder). This opens the possibility of real time high resolution images of the retina with WFC with robust devices and low cost.

In sum, the answer to the question: Is WFC an alternative to AO for retinal Imagen? We believe that the answer is "Yes it is!”

\section{Acknowledgments}

This work was supported by the Xunta de Galicia , grant EM2013/030, the Spanish ministry of Economía y Competitividad grant FIS2013-46188-P and FIS2012-38244C02-01

\section{References}

[1] Fernandez, E. J., Iglesias, I., \& Artal, P., "Closed3-loop adaptive optics in the human eye”. Optics Letters, 26(10), 746-748 (2001).

[2] D. Catlin, C. Dainty, "High resolution imaging of the human retina with a Fourier deconvolution technique," J.Opt.Soc.Am. A 19(8), 1515-1523 (2002).

[3] V Nourrit, B. Vohnsen, P. Artal, "Blind deconvolution for highresolution confocal scanning laser ophthalmoscopy,” J. Opt. A: Pure Appl. Opt. 7, 585-592 (2005)

[4] E. R. Dowski, Jr., and W. T. Cathey, "Extended depth of field through wave-front coding," Appl. Opt. 34, 1859-1866 (1995).

[5] E. Acosta, J. Arines, "Optical-digital System Invariant to eye aberrations for retinal imaging”, ARVO2012

[6] J. Arines, Partially compensated deconvolution from wavefront sensing images of the eye fundus, Opt. Comm.Volume 284, Issue 6,284(6), 1548-1552 (2011)

[7] J. Arines, R. O. Hernandez, S. Sinzinger, A. Grewe, and E. Acosta, Wavefront-coding technique for inexpensive and robust retinal imaging, Opt. Lett. 39, 3986-3988 (2014).

[8] L. N. Thibos, X. Hong, A. Bradley, and X. Cheng, Statistical variation of aberration structure and image quality in a normal population of healthy eyes, J. Opt. Soc. Am. A 19, 2329-2348 (2002). 